\title{
LIFETIME MODELLING AT APS AND ESRF
}

\author{
A. Ropert, ESRF, BP 220, F - 38043 Grenoble Cedex
}

\section{Abstract}

Achieving long lifetimes is a major concern for all third generation light sources, even for high energy rings like the APS $(7 \mathrm{GeV})$ or the ESRF $(6 \mathrm{GeV})$. Due to the small electron transverse dimensions and the trend towards small gap undulator vacuum vessels, Touschek scattering and gas scattering make signficant contributions to the lifetime reduction. Taking action to improve the lifetime therefore requires a reliable lifetime model. The strategy used on both machines to decouple the different contributions to the lifetime is presented and the experimental results analysed. Practical limitations on the lifetime modelling are discusssed.

\section{INTRODUCTION}

APS and ESRF are two third generation light sources optimized for the production of hard X-rays. Among the processes that limit the lifetime, the gas scattering lifetime reduction due to small gap undulator chambers (leading to a beam stay-clear of $5 \mathrm{~mm}$ at APS, $8 \mathrm{~mm}$ at the ESRF) and the enhanced Touschek scattering induced by the increased density in the bunch volume (horizontal emittance in the few nanometer range, coupling in the 1 $\%$ range) are the more critical.

Table1: APS and ESRF relevant parameters

\begin{tabular}{|c|c|c|}
\hline & APS & ESRF \\
\hline Energy & 7 & 6 \\
\hline Particles & $\mathrm{e}^{+}($in 1998$)$ & $\mathrm{e}^{-}$ \\
\hline Average $\beta_{\mathrm{v}}$ & 15.76 & 20.74 \\
\hline Transverse acceptance m.rad & $9.2510^{-7}$ & $3.210^{-6}$ \\
\hline Horizontal emittance $\quad \mathrm{nm}$ & 8.6 & 3.8 \\
\hline Coupling & 1.6 & 0.7 \\
\hline Standard filling pattern & $6+25 * 3$ & $\begin{array}{r}662 \\
\end{array}$ \\
\hline Lifetime & $30 @ 100 \mathrm{~mA}$ & 48@200mA \\
\hline RF voltage & 9.5 & $\begin{array}{l}11.67 \\
\end{array}$ \\
\hline Natural bunch length & 8.5 & 3.7 \\
\hline Bunch lengthening & 1.6 & 1 \\
\hline Energy acceptance & 1.7 & 2.5 \\
\hline \begin{tabular}{|ll} 
Average pressure & $\mathrm{nT}$ \\
\end{tabular} & 0.5 & 1 \\
\hline \begin{tabular}{|ccc} 
Gas composition & $\mathrm{H}_{2}$ & $\%$ \\
& $\mathrm{CH}_{4}$ & \\
& $\mathrm{H}_{2} \mathrm{O}$ & \\
& $\mathrm{CO}$ & \\
& $\mathrm{CO}_{2}$ & \\
\end{tabular} & $\begin{array}{c}66 \\
7 \\
2 \\
21 \\
4 \\
\end{array}$ & $\begin{array}{l}6.5 \\
0.5 \\
\end{array}$ \\
\hline
\end{tabular}

As shown in Table 1, many parameters play a role in the modelling of the different contributions to the lifetime. Since the 2 rings are Touschek lifetime dominated, good lifetime modelling requires dedicated experimental conditions in order to decouple the different contributions and to minimize the uncertainties on measured parameters [1], [2].

\section{ELASTIC GAS SCATTERING LIFETIME}

\subsection{Measuring technique}

Measurements were focused on the vertical gas scattering contribution which is the dominant effect (for instance, the horizontal acceptance is 4 times larger than the vertical one at ESRF).

The procedure consists in imposing an additional aperture reduction by progressively closing one jaw (upper or lower) of a vertical scraper and recording the lifetime evolution versus the scraper aperture at different beam intensities.

In order to minimize the Touschek contribution, the machines were operated in the following conditions:

i) filling pattern with a large number of bunches $(83 *$ 10 for APS, 662 for ESRF) to get a small bunch current

ii) large coupling obtained by bringing the tunes closer to the coupling resonance (APS) or by detuning a skew quadrupole corrector (ESRF)

Simulations and measurements are summarized in Fig. 1 and Fig. 2. In the APS case for instance, the interesting zone for the scraper stands between $0.65 \mathrm{~mm}$ (quantum limit) and $2 \mathrm{~mm}$ (above this aperture, the contribution of the other lifetimes starts to be too large).

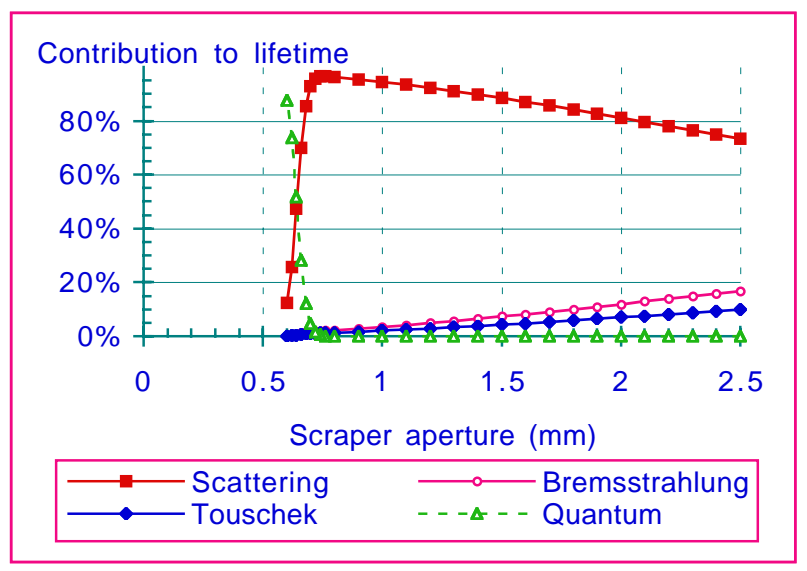

Figure 1: Evolution of the different lifetime contributions as a function of scraper aperture 


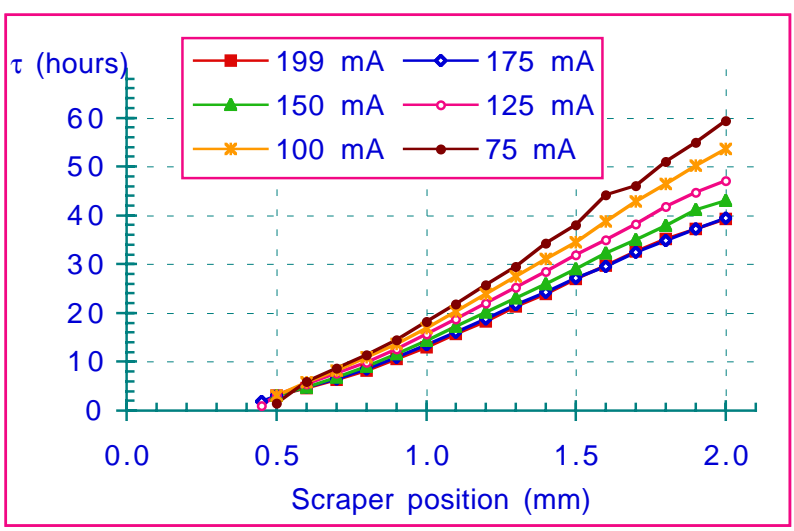

Figure 2: ESRF lifetime as a function of scraper position

\subsection{Data analysis}

The analysis of measurements is based on the expected dependence of the elastic gas scattering lifetime $\tau_{\mathrm{G}}$ on the vertical acceptance $A_{y}$ since:

$$
\frac{1}{\tau_{\mathrm{G}}}=\mathrm{C}_{\mathrm{G}} \frac{\beta_{\mathrm{A}}}{\mathrm{A}_{\mathrm{y}}^{2}} \sum_{\text {atomsj }}\left[\mathrm{Z}_{\mathrm{j}}^{2} \sum_{\text {gasi }} \alpha_{\mathrm{ij}}<\beta_{\mathrm{y}} \mathrm{P}_{\mathrm{i}}>\right]
$$

with $C_{G}$ being a constant and $\beta_{A}$ the $\beta$ function at the location where the acceptance is limited. $\tau_{\mathrm{G}}$ also depends on the gas composition which is defined by the residual pressure $\mathrm{P}_{\mathrm{i}}$, the atomic number $\mathrm{Z}_{\mathrm{j}}$ and the number of atoms $\alpha_{i j}$ of the different species.

Therefore $\frac{1}{\tau_{\text {meas }}}$ can be fitted to a linear law $\mathrm{b}+\mathrm{aA}_{\mathrm{y}}^{-2}$, where $\mathrm{b}$ accounts for scraper independent lifetime contributions. Using the same analysis for the different beam currents yields the gas scattering loss rate $\frac{1}{\tau_{\mathrm{G}}}=\frac{\beta_{\mathrm{ID}}}{\beta_{\mathrm{A}_{\mathrm{y}}}} \frac{\mathrm{a}}{\mathrm{A}_{\mathrm{ID}}^{2}}=\mathrm{cI}+\mathrm{d}\left(\beta_{\mathrm{ID}}\right.$ and $\mathrm{A}_{\mathrm{ID}}$ are the vertical $\beta$ and the aperture at ID location) and the pressure law $\mathrm{P}=\mathrm{P}_{0}+\frac{\partial \mathrm{P}}{\partial \mathrm{I}} \mathrm{I}\left(\mathrm{P}_{0}\right.$ being the static pressure and $\frac{\partial \mathrm{P}}{\partial \mathrm{I}}$ the desorption coefficient). Table 2, Fig. 3 and Fig. 4 illustrate the results of this analysis.

Table 2: Results of the fitting procedure

\begin{tabular}{|c|c|c|}
\hline & APS & ESRF \\
\hline Loss rate $\left(\right.$ hours $\left.^{-1} \cdot \mathrm{mA}^{-1}\right)$ & $1.5510^{-5}$ & $1.4410^{-5}$ \\
\hline Desorption (Torr.mA ${ }^{-1}$ ) & $2.9410^{-12}$ & $1.9610^{-11}$ \\
\hline
\end{tabular}

\subsection{Modelling limitations and credibility}

Any error in the position of the scraper with respect to the beam axis affects the analysis of raw data. In order to take into account alignment or displacement errors, an offset has to be introduced in the fit of the measured lifetimes. The error bars in Fig. 3 correspond to a $\pm 25 \mu \mathrm{m}$ uncertainty on the scraper position.

In both machines, a significant discrepancy between measured and predicted pressures is observed. Several explanations can be advanced: non-ideal location and/or calibration of gauges, imprecision in the knowledge of the residual gas composition, long time constant for the pressure to get stabilized, importance of the weighting of the pressures by local $\beta$-functions. At ESRF, the moderate reproducibility of measurements linked to nonidentical vacuum conditions (variation of the loss rate between 1.44 and $2.4210^{-5}$ hours $^{-1} \cdot \mathrm{mA}^{-1}$ ) also illustrates the difficulties in obtaining good modelling.

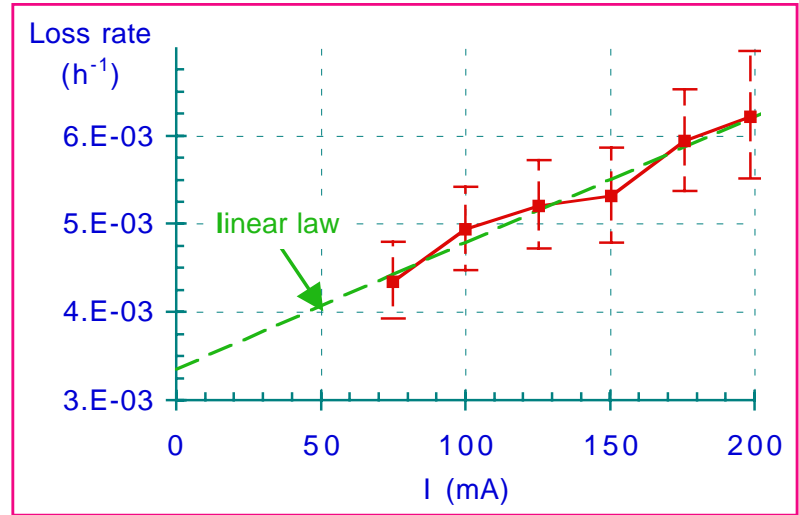

Figure 3: ESRF loss rate as a function of current

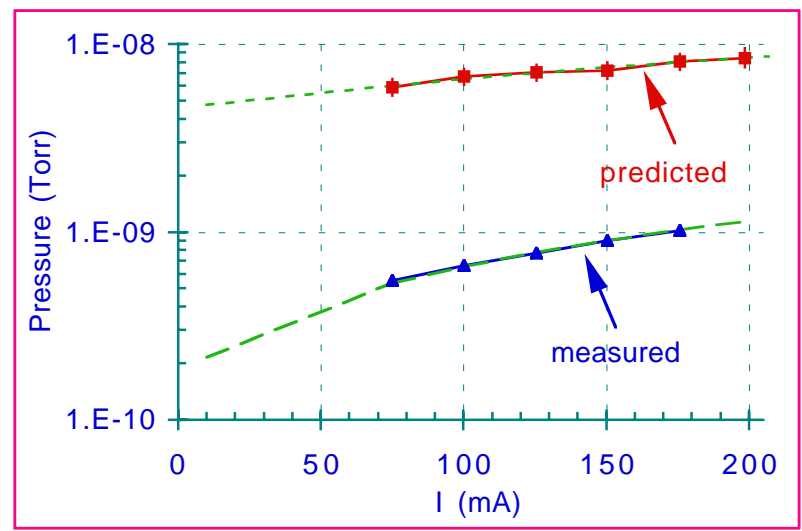

Figure 4: ESRF average pressure as a function of current

\section{TOUSCHEK LIFETIME}

The Touschek lifetime can be expressed as:

$$
\frac{1}{\tau_{\mathrm{T}}}=\frac{\mathrm{Nr}_{0}^{2} \mathrm{c}}{8 \pi \sigma_{\mathrm{x}} \sigma_{\mathrm{y}} \sigma_{\mathrm{L}} \beta^{3} \gamma^{2}\left(\frac{\Delta \mathrm{p}}{\mathrm{p}}\right)^{3}} \mathrm{f}\left(\frac{\Delta \mathrm{p}}{\mathrm{p}}, \sigma_{\mathrm{x}}\right)
$$

where $\mathrm{N}$ is the number of particles per bunch, $\sigma_{\mathrm{x}}$ and $\sigma_{y}$ the transverse dimensions, $\sigma_{\mathrm{L}}$ the bunch length and $(\Delta \mathrm{p} / \mathrm{p})$ the energy acceptance which is determined by either the RF bucket or by transverse limitations (physical or dynamic aperture).

An almost pure Touschek lifetime is obtained when operating in single bunch mode at low coupling. Since the only unknown parameter is the energy acceptance, the strategy consists in recording the lifetime evolution as a function of the RF voltage and in deducing $(\Delta p / p)$ from the fit of the experimental data. 
The results of APS (5.4 mA, coupling $\geq 1.6 \%)$ and ESRF ( $3.5 \mathrm{~mA}, 0.77 \%$ coupling) are shown in Fig. 5 and Fig. 6 and compared with simulations.

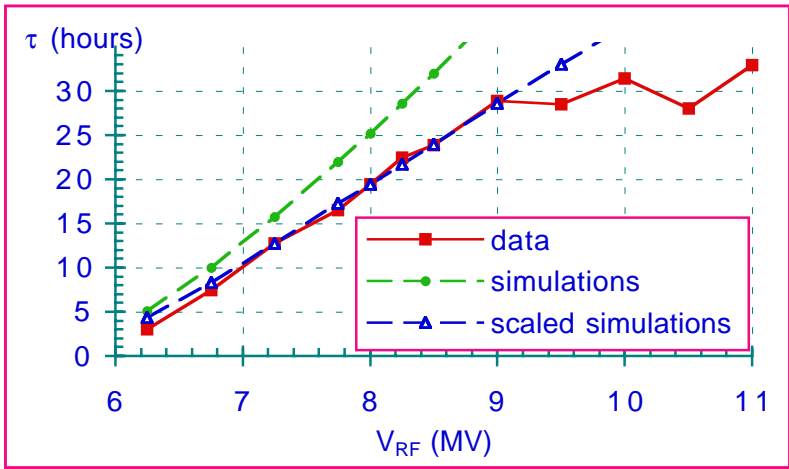

Figure 5: Lifetime evolution versus RF voltage at APS

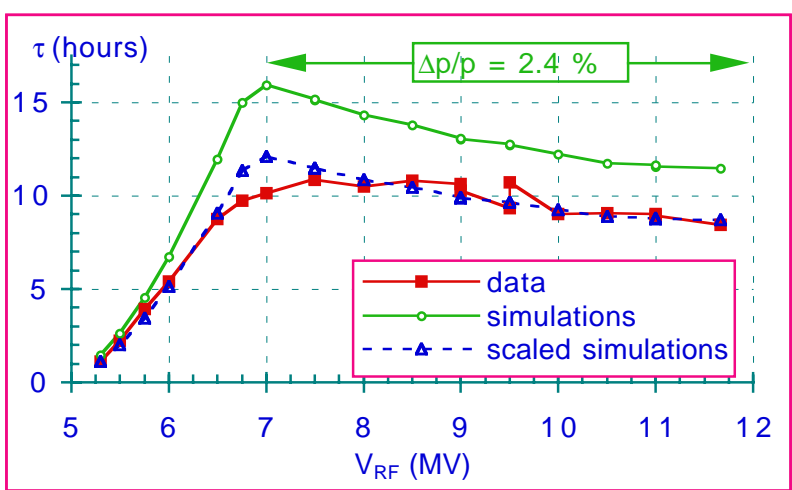

Figure 6: Lifetime evolution versus RF voltage at ESRF

In both machines, the asymptotic behaviour of the lifetime above a given RF voltage clearly shows that the limitation of the energy acceptance is dictated by transverse considerations and not by the available RF voltage.

The main sources of uncertainties come from the measured transverse emittances and bunch lengths since, for both machines, the bunch volume has to be scaled down (a factor 0.88 at APS and 0.76 at ESRF) in order to arrive at a good agreement between predicted and measured lifetimes.

\section{BREMSSTRAHLUNG LIFETIME}

The modelling of the Bremsstrahlung contribution is the most delicate since it is never the dominant contribution to the lifetime.

At ESRF, the method used was to record the evolution of the lifetime versus the RF voltage in conditions where the Touschek contribution is minimized (uniform filling, low current, large coupling). In addition to time consuming lifetime measurements, the already mentioned uncertainties on pressure, transverse beam sizes,.. make the comparison with simulations very difficult.

At APS, the lifetime evolution was recorded as a function of the current (Fig. 7) for different filling patterns $(83 * 10,83 * 2,25 * 3$ and $22 * 1$ bunches).

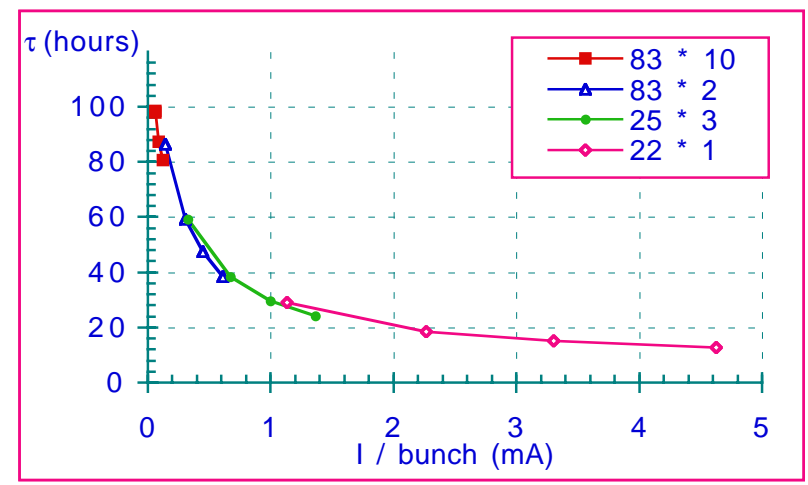

Figure 7: Lifetime dependence on bunch current at APS

Since the assumption of constant Bremsstrahlung and gas scattering contributions at a given current, whatever the filling pattern, looks reasonable, one can write:

$$
\frac{1}{\tau_{\text {meas }}}=\mathrm{a}+\frac{1}{\tau_{\mathrm{T}}}=\mathrm{a}+\mathrm{b} \frac{\mathrm{I}_{\text {bunch }}}{\sigma_{\mathrm{L}}\left(\mathrm{I}_{\text {bunch }}\right)}=\mathrm{a}+\mathrm{cI}_{\text {bunch }}^{\frac{2}{3}}
$$

using the dependence of $\frac{1}{\tau_{\mathrm{T}}}$ on the bunch current and the dependence of the bunch length on the bunch current which is given by:

$$
\left[\frac{\sigma_{\mathrm{L}}\left(\mathrm{I}_{\text {bunch }}\right)}{\sigma_{\mathrm{L}}\left(\mathrm{I}_{\text {bunch }}=0\right)}\right]^{3}-\frac{\sigma_{\mathrm{L}}\left(\mathrm{I}_{\text {bunch }}\right)}{\sigma_{\mathrm{L}}\left(\mathrm{I}_{\text {bunch }}=0\right)}=\mathrm{kI}_{\text {bunch }}
$$

The fit of the measured lifetimes to the above analytical expression is excellent. The elastic and inelastic gas scattering loss rate can be estimated at: $\frac{1}{\tau_{\mathrm{G}}}+\frac{1}{\tau_{\mathrm{B}}}=4.7510^{-5}$ hours $^{-1} \cdot \mathrm{mA}^{-1}$.

\section{CONCLUSIONS}

The transverse-related limitation of the APS and ESRF energy acceptance was unambiguously established. The reasons for this are under investigation. The enlargement of the energy acceptance could significantly increase lifetime performance in the few bunch mode.

The strategy for modelling the gas scattering lifetime looks adequate but requires stable vacuum conditions to be fully exploited.

\section{ACKNOWLEDGMENTS}

I am very grateful to J. Galayda for giving me the opportunity of participating in the APS machine studies. I also want to thank the kind assistance of M. Borland, L. Emery and N. Sereno at APS, L. Farvacque at ESRF during the measurements.

\section{REFERENCES}

[1] A. Ropert, APS Light Source Note, Sept 1998

[2] A. Ropert, ESRF Notes (74-96/MDT, 53-98/MDT, 08-99/MDT) 